OPEN ACCESS

Edited by:

Paolo De Fabritiis,

University Tor Vergata, Italy

Reviewed by:

Massimiliano Bonifacio,

University of Verona, Italy

Elisabetta Abruzzese,

University of Rome Tor Vergata, Italy

*Correspondence:

Daniele Cattaneo

daniele.cattaneo@unimi.it

Specialty section:

This article was submitted to

Hematologic Malignancies,

a section of the journal

Frontiers in Oncology

Received: 20 November 2021

Accepted: 16 December 2021

Published: 12 January 2022

Citation:

Barozzi E, Bucelli C, Grifoni Fl, Gianelli U, lurlo $A$ and Cattaneo D

(2022) Successful Imatinib

Treatment for Systemic Mastocytosis

Associated With Myelodysplastic/

Myeloproliferative Neoplasm: Report of a Case and Literature Review.

Front. Oncol. 11:819097.

doi: 10.3389/fonc.2021.819097

\section{Successful Imatinib Treatment for Systemic Mastocytosis Associated With Myelodysplastic/ Myeloproliferative Neoplasm: Report of a Case and Literature Review}

\author{
Enrico Barozzi ${ }^{1,2}$, Cristina Bucelli ${ }^{1}$, Federica Irene Grifoni ${ }^{1}$, Umberto Gianelli ${ }^{3,4}$, \\ Alessandra lurlo ${ }^{1}$ and Daniele Cattaneo ${ }^{1,2 *}$ \\ 1 Hematology Division, Foundation IRCCS Ca' Granda Ospedale Maggiore Policlinico, Milan, Italy, ${ }^{2}$ Department of Oncology \\ and Hemato-Oncology, University of Milan, Milan, Italy, ${ }^{3}$ Department of Pathophysiology and Transplantation, University of \\ Milan, Milan, Italy, ${ }^{4}$ Division of Pathology, Foundation IRCCS Ca' Granda Ospedale Maggiore Policlinico, Milan, Italy
}

Systemic mastocytosis (SM) is a heterogeneous disease characterized by the expansion of mast cells in one or more tissues, frequently characterized by the presence of KITD816V mutation. The updated World Health Organization (WHO) classification of myeloid neoplasms recognizes SM with an associated hematological neoplasm (SM$\mathrm{AHN}$ ) as a new subtype among the others, which is depicted by the coexistence of SM with another hematological clonal disease. Prognosis is very different among SM patients, while its treatment, although highly personalized, is still challenging. Here we report a case of KITD816V-unmutated SM associated with MDS/MPN successfully treated with imatinib.

Keywords: imatinib, systemic mastocytosis, SM-AHN, MDS/MPN, myeloid neoplasms

\section{INTRODUCTION}

Systemic mastocytosis (SM) is a rare hematological neoplasm characterized by the abnormal proliferation and accumulation of mast cells (MCs). Clinical manifestations are heterogeneous depending on the tissue infiltration and MC mediators released by their degranulation (1). Minor diagnostic criteria include: (I) $>25 \%$ of all MCs are atypical cells on bone marrow (BM) smears; (II) gainof-function point mutations at codon 816 of KIT gene; (III) high serum tryptase level (unless there is an associated clonal myeloid disorder, in which case this parameter is not valid); and (IV) abnormal MCs CD25/CD2 expression. However, the unique major criterion is depicted by multifocal, dense infiltrates of MCs ( $\geq 15$ MCs in aggregates) detected in sections of BM and/or other extracutaneous organs $(2,3)$. Accordingly, the diagnosis of SM can be made when the major criterion and one minor criterion or at least three minor criteria are present. In the WHO classification, SM is divided into: indolent SM; smoldering SM (SSM); SM with an associated hematological neoplasm (SM-AHN); aggressive SM; and MC leukemia (2,3). Signs of an excessive MC burden in the tissue, called B-findings, and signs of specific organ damage, called C-findings, are used to define the different subgroups of SM (4). Regarding SM$\mathrm{AHN}$, whose diagnosis requires the presence of both SM criteria and WHO criteria for a clonal 
hematological neoplasm, SM is most frequently associated with myeloid malignancies such as myeloproliferative neoplasms (MPN), myelodysplasia (MDS), and MDS/MPN overlapping syndromes, such as chronic myelomonocytic leukemia (CMML). The goal of SM therapy is stratified according to the specific subtype. Indolent SM therapy is focused on symptom relief such as pruritus, flushing, gastrointestinal cramping, and osteoporosis, while in the aggressive forms the primary therapeutic target is the improvement of organ damage by means of cytoreductive and/or targeted therapies, including tyrosine-kinase inhibitors $(1,4-6)$.

\section{CASE DESCRIPTION}

A 56-year-old Caucasian woman presented to our hospital with maculopapular rash of the chest, hepato-splenomegaly, and enlarged inguinal, axillary, and lateral cervical lymph nodes with a short axis less than $1 \mathrm{~cm}$. Importantly, the patient suffered from different antibiotic allergies (namely, penicillin and vancomycin). Past medical history was remarkable for breast carcinoma treated in 2007 with radiotherapy and hormone therapy. In addition, two years before the first access to our hospital, the patient suffered from vertebral fracture initially interpreted as post-traumatic. Routine blood analysis revealed mild anemia ( $\mathrm{Hb} 10.5 \mathrm{~g} / \mathrm{dl}$ ), white blood cells count $(\mathrm{WBC})$ of $12.3 \times 10^{9} / \mathrm{L}$ with significantly increased basophils $\left(3.08 \times 10^{9} / \mathrm{L}\right)$, and thrombocytosis (platelets count, $567 \times$ $\left.10^{9} / \mathrm{L}\right)$. Serum lactate dehydrogenase (LDH) level was $224 \mathrm{IU} / \mathrm{L}$. Renal function tests were normal, and also coagulation, C-reactive protein, and liver function tests (including alkaline phosphates). Neither folic acid nor cobalamin deficiency was detected. The abdomen ultrasonography showed an enlarged spleen (diameter of $19 \mathrm{~cm}$ ), while enlarged lymph nodes were defined as reactive. Screening for JAK2V617F, CALR, and MPL mutations, and BCR$A B L 1$ p210, p190, and p230 fusion transcripts were all negative. Therefore, a BM aspirate and biopsy were performed, the first with characteristics of dyserithropoiesis with megaloblastic changes and of dismegakaryopoiesis with hypolobated and multinucleated megakaryocytes. BM examination revealed a myeloid/erythroid ratio of $>5 / 1$, and grade 2 reticulin fibrosis, loose and dense clusters of hyperlobulated or hypolobulated and multinucleated megakaryocytes, along with an infiltrate of approximately $30 \%$ of atypical MCs, which were tryptase+, CD117+/-, CD25+/-, and CD30-. Cytogenetic analysis revealed a normal female karyotype. Next-generation-sequencing test for myeloid genes (Illumina MiSeqTM) detected no mutations in any of the 30 genes analyzed, including KIT. Second-level analyses showed increased serum tryptase level (148 ng/ml) and peripheral CD34+ cells (172/ $\mu \mathrm{l})$. Thus, a diagnosis of SM with associated MDS/MPN, unclassifiable (MDS/MPN, U) was made fitting the major criterion and one minor criterion for SM and the clinical, morphological and molecular WHO criteria for MDS/MPN, U. Dual-energy X-ray absorptiometry (DEXA) showed lumbar TScore: -1.5 , and total femur and femoral neck (total: +0.2 , femoral neck: -1.3 ) were suggestive for osteopenia that is a typical finding of SM. Since the search for KITD816V mutation was negative, imatinib $400 \mathrm{mg}$ daily was started. After 2 months of therapy, the patient achieved significant improvement in hematological parameters, namely, $\operatorname{WBC}\left(7.17 \times 10^{9} / \mathrm{L}\right)$ and platelets $\left(177 \times 10^{9} / \mathrm{L}\right)$, along with complete resolution of the skin rash. $\mathrm{LDH}$ level decreased to $136 \mathrm{IU} / \mathrm{L}$, and tryptase to $3 \mathrm{ng} / \mathrm{ml}$. Due to grade 3 thrombocytopenia imatinib was suspended for two weeks. When platelet count recovered (greater than $100 \times 10^{9} / \mathrm{L}$ ), imatinib was resumed at a lower dose (initially $100 \mathrm{mg}$ daily, and then $200 \mathrm{mg}$ daily). After 3 months of therapy, a new BM biopsy was performed, showing an outstanding response of SM and an improvement in both MDS/MPN and BM fibrosis grade (MF-1) (Figure 1). Mast cells decreased up to 1-2\%, serum tryptase level on peripheral blood remained within normal range and spleen diameter decreased up to $16 \mathrm{~cm}$. At last follow-up, imatinib was still well tolerated except for mild fluid retention, with no more episodes of hematological toxicity.

\section{DISCUSSION}

Systemic mastocytosis is a heterogeneous hematological neoplasm ranging from indolent to aggressive forms with different prognoses. According to the updated WHO classification, the category of SM-AHN represents a very complex SM variant, depicted by the simultaneous occurrence of hematological clonal non-MC lineage diseases, more frequently myeloid neoplasms in about $85-90 \%$ of cases $(2,7)$.

Although its prognosis depends on both the SM variant and the associated disease, SM-AHN is generally considered to be an advanced variant, multilineage-mutated myeloid neoplasia with a fatal outcome. It is also often difficult to accurately relate the $\mathrm{B} /$ $\mathrm{C}$ findings to $\mathrm{SM}$ or associated disease, and thus the correct classification of the SM variant (8).

Here, we describe the case of a 56-year-old Caucasian woman with KITD816V-unmutated SM associated with a myeloid neoplasm showing overlapping features of both MDS and BCRABL1-negative MPN. In detail, BM biopsy sample was hypercellular with increased granulopoiesis and loose and dense clusters of megacaryocytes with MPN- and MDS-like characteristics. Additionally, along with splenomegaly, complete blood cells examination showed basophilic leukocytosis with thrombocytosis and mild anemia, allowing us to make a diagnosis of SM associated with MDS/MPN, U versus the alternative option of SSM.

The pathophysiologic basis of the coexistence of SM and other myeloid neoplasms is poorly understood; notably, the driver KIT mutation may not be present in the concurrent neoplasm (7). Sotlar et al. investigated the presence of KITD816V mutation in the AHN, showing the coexistence of this mutation in $89 \%$ of cases associated with CMML, but only in $20 \%$ of patients with SM-MPN (9). Gainof-function KIT mutations lead to a ligand-independent activation of downstream signaling involving PI3K/AKT/mTOR, JAK/STAT, and RAS/RAF/MEK/ERK pathways, promoting proliferation and resistance to apoptosis (10). Non-codon-816 KIT mutations have been detected in both patients affected by cutaneous mastocytosis and SM, and they may facilitate the transformation into an aggressive form (3). Overall, KIT mutations are detected in over $80 \%$ of all SM, nevertheless, additional somatic mutations are reported especially in SM-AHN, including TET2, SRSF2, ASXL1, $R U N X 1, J A K 2$, and RAS mutations which may impact on survival, 

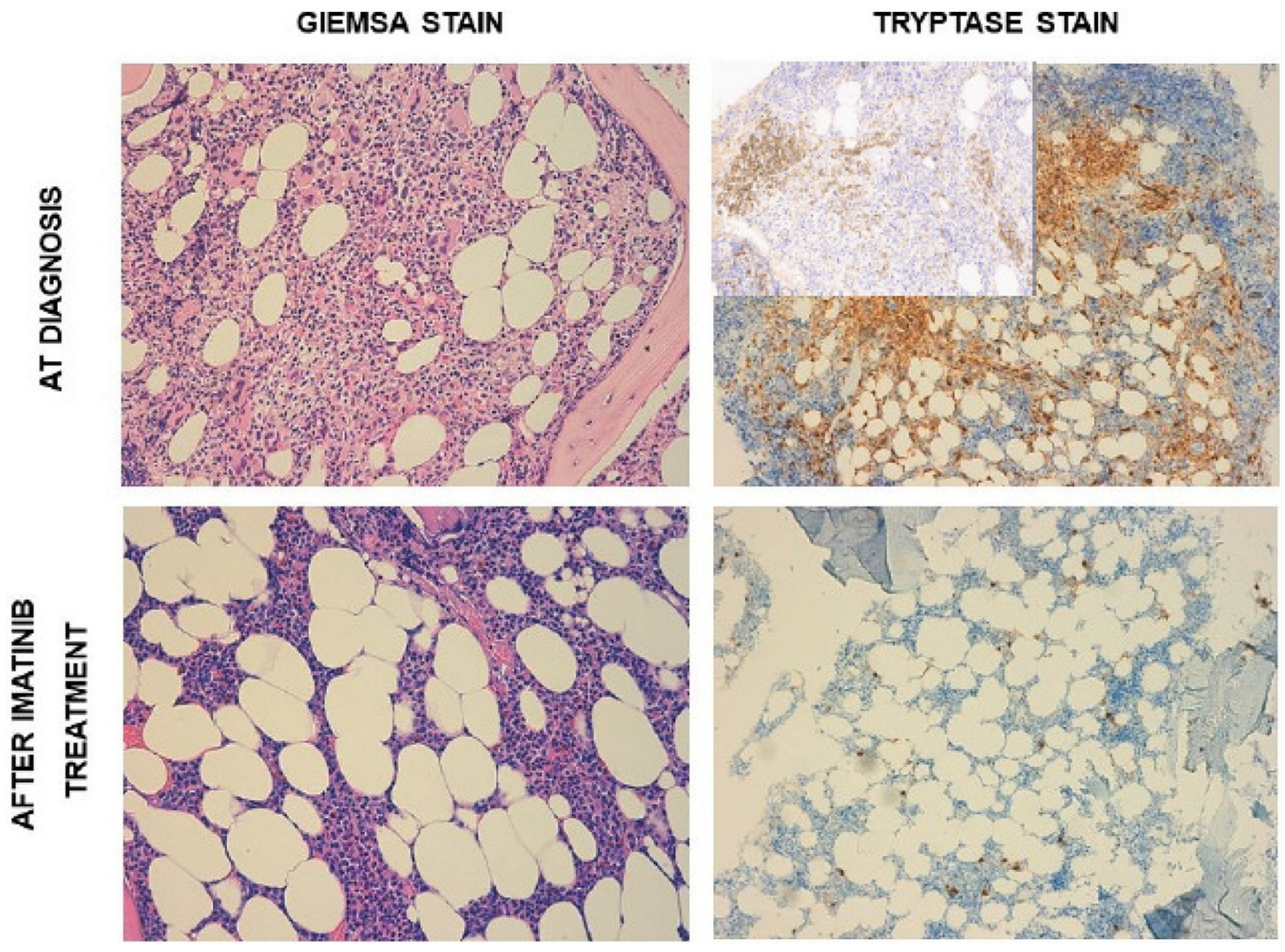

FIGURE 1 | Upper quadrants: bone marrow morphology at diagnosis. Lower quadrants: bone marrow morphology after imatinib treatment (3 months). Left quadrants: Giemsa stain. Right quadrants: tryptase stain with CD117 immunohistochemistry (insert).

leading to a better prognostication and new targeted treatment in SM (11). Several cases with these somatic mutations have been reported in SM associated with MDS/MPN, showing a very dissimilar prognosis (12). A major challenge is how to integrate clinical characteristics, morphology, and genetic to clarify the diagnosis and prognosis of complex SM subtypes (13). Therefore, treatment in SM is highly individualized (1). Nevertheless, their management could be very difficult, and SM-AHN may transform into more aggressive forms (14). KIT mutations are a compass and often guide the therapeutic choice. Since our patient did not show KITD816V mutation, treatment with imatinib was started. Indeed, imatinib has already demonstrated high efficacy in vitro, inhibiting both wild-type KIT and juxtamembrane mutant c-kit kinase activity, but has no effect on D816V mutant variant (15). In a phase II trial 20 patients regardless their KIT mutational status were treated with imatinib, however only one KITD816V-negative patient achieved complete remission, with other six patients showing symptoms improvement, among them two patient were KITD816V-positive (16). Altogether, these data suggest that KITD816V mutation is associated with resistance to imatinib, while, as in our patient, it could represent a suitable approach in KITD816V-unmutated cases, even in those associated with MDS/MPN.

\section{DATA AVAILABILITY STATEMENT}

The original contributions presented in the study are included in the article/supplementary material. Further inquiries can be directed to the corresponding author.

\section{ETHICS STATEMENT}

Written informed consent was obtained from the individual(s) for the publication of any potentially identifiable images or data included in this article.

\section{AUTHOR CONTRIBUTIONS}

All authors listed have made a substantial, direct, and intellectual contribution to the work and approved it for publication.

\section{FUNDING}

The only funds used were those provided by the authors' institutions. 


\section{REFERENCES}

1. Pardanani A. Systemic Mastocytosis in Adults: 2021 Update on Diagnosis, Risk Stratification and Management. Am J Hematol (2021) 96:508-25. doi: 10.1002/ajh.26118

2. Arber DA, Orazi A, Hasserjian R, Thiele J, Borowitz MJ, Le Beau MM, et al. The 2016 Revision to the World Health Organization Classification of Myeloid Neoplasms and Acute Leukemia. Blood (2016) 127:2391-405. doi: 10.1182/blood-2016-03-643544

3. Valent P, Akin C, Hartmann K, Nilsson G, Reiter A, Hermine O, et al. Advances in the Classification and Treatment of Mastocytosis: Current Status and Outlook Toward the Future. Cancer Res (2017) 77:1261-70. doi: 10.1158/ 0008-5472.CAN-16-2234

4. Leguit R, Hebeda K, Kremer M, van der Walt J, Gianelli U, Tzankov A, et al. The Spectrum of Aggressive Mastocytosis: A Workshop Report and Literature Review. Pathobiology (2020) 87:2-19. doi: 10.1159/000504099

5. Carter MC, Metcalfe DD, Komarow HD. Mastocytosis. Immunol Allergy Clinics North America (2014) 34:181-96. doi: 10.1016/j.iac.2013.09.001

6. Abid A, Malone MA, Curci K. Mastocytosis. Primary Care: Clinics Office Pract (2016) 43:505-18. doi: 10.1016/j.pop.2016.04.007

7. Valent P, Horny HP, Escribano L, Longley BJ, Li CY, Schwartz LB, et al. Diagnostic Criteria and Classification of Mastocytosis: A Consensus Proposal. Leuk Res (2001) 25:603-25. doi: 10.1016/S0145-2126(01)00038-8

8. Zanotti R, Tanasi I, Crosera L, Bonifacio M, Schena D, Orsolini G, et al. Systemic Mastocytosis: Multidisciplinary Approach. Mediterr J Hematol Infect Dis (2021) 13:e2021068. doi: 10.4084/MJHID.2021.068

9. Sotlar K, Colak S, Bache A, Berezowska S, Krokowski M, Bültmann B, et al. Variable Presence of KITD816V in Clonal Haematological non-Mast Cell Lineage Diseases Associated With Systemic Mastocytosis (SM-AHNMD). J Pathol (2010) 220:586-95. doi: 10.1002/path.2677

10. Gilreath J, Tchertanov L, Deininger M. Novel Approaches to Treating Advanced Systemic Mastocytosis. CPAA (2019) 11:77-92. doi: 10.2147/ CPAA.S206615

11. Ustun C, Arock M, Kluin-Nelemans HC, Reiter A, Sperr WR, George T, et al. Advanced Systemic Mastocytosis: From Molecular and Genetic Progress to Clinical Practice. Haematologica (2016) 101:1133-43. doi: 10.3324/ haematol.2016.146563
12. Mimiola E, Bomben R, De Matteis G, Perbellini O, Guglielmelli P, Bonifacio M, et al. Systemic Mastocytosis Associated With Myelodysplastic/Myeloproliferative Neoplasms With Ring Sideroblasts and Thrombocytosis: Report of Three Cases. Hematol Oncol (2019) 37:628-33. doi: 10.1002/hon.2680

13. Reiter A, George TI, Gotlib J. New Developments in Diagnosis, Prognostication, and Treatment of Advanced Systemic Mastocytosis. Blood (2020) 135:1365-76. doi: 10.1182/blood.2019000932

14. Grifoni FI, Sciumè M, Pravettoni V, Ulivieri FM, Muratori S, Fracchiolla NS, et al. A Case Report of Systemic Mastocytosis Associated With Multiple Hematologic Non-Mast Cell Lineage Diseases. Hematol Oncol (2019) 37:20511. doi: $10.1002 /$ hon.2605

15. Zermati Y, De Sepulveda P, Féger F, Létard S, Kersual J, Castéran N, et al. Effect of Tyrosine Kinase Inhibitor STI571 on the Kinase Activity of WildType and Various Mutated C-Kit Receptors Found in Mast Cell Neoplasms. Oncogene (2003) 22:660-4. doi: 10.1038/sj.onc.1206120

16. Vega-Ruiz A, Cortes JE, Sever M, Manshouri T, Quintás-Cardama A, Luthra $\mathrm{R}$, et al. Phase II Study of Imatinib Mesylate as Therapy for Patients With Systemic Mastocytosis. Leuk Res (2009) 33:1481-4. doi: 10.1016/ j.leukres.2008.12.020

Conflict of Interest: The authors declare that the research was conducted in the absence of any commercial or financial relationships that could be construed as a potential conflict of interest.

Publisher's Note: All claims expressed in this article are solely those of the authors and do not necessarily represent those of their affiliated organizations, or those of the publisher, the editors and the reviewers. Any product that may be evaluated in this article, or claim that may be made by its manufacturer, is not guaranteed or endorsed by the publisher.

Copyright (c) 2022 Barozzi, Bucelli, Grifoni, Gianelli, Iurlo and Cattaneo. This is an open-access article distributed under the terms of the Creative Commons Attribution License (CC BY). The use, distribution or reproduction in other forums is permitted, provided the original author(s) and the copyright owner(s) are credited and that the original publication in this journal is cited, in accordance with accepted academic practice. No use, distribution or reproduction is permitted which does not comply with these terms. 